\title{
A New Age and Distance for I Zw 18, the Most Metal-Poor Galaxy in the Nearby Universe
}

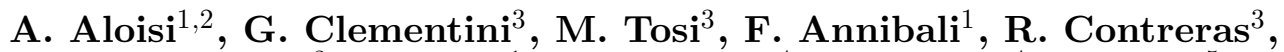 \\ G. Fiorentino ${ }^{3}$, J. Mack $^{1}$, M. Marconi ${ }^{4}$, I. Musella ${ }^{4}$, A. Saha ${ }^{5}$, \\ M. Sirianni ${ }^{1,2}$, and R. P. van der Marel $^{1}$ \\ ${ }^{1}$ Space Telescope Science Institute, \\ 3700 San Martin Drive, Baltimore, MD 21218, USA \\ email: aloisi@stsci.edu \\ ${ }^{2}$ On assignment from the Space Telescope Division of the European Space Agency \\ ${ }^{3}$ INAF-Osservatorio Astronomico di Bologna, \\ Via Ranzani 1, I-40127 Bologna, Italy \\ ${ }^{4}$ INAF-Osservatorio Astronomico di Capodimonte, \\ Via Moiariello 16, I-80131 Napoli, Italy \\ ${ }^{5}$ National Optical Astronomy Observatory, \\ P.O. Box 26732, Tucson, AZ 85726
}

\begin{abstract}
The blue compact dwarf galaxy I Zw 18 holds the record of the lowest metallicity ever observed in the local universe. As such, it represents the closest analog to primordial galaxies in the early universe. More interestingly, it has recurrently been regarded as a genuinely young galaxy caught in the process of forming in the nearby universe. However, stars of increasingly older ages are found within I Zw 18 every time deeper high-resolution photometric observations are performed with the Hubble Space Telescope (HST): from the original few tens of Myrs to, possibly, several Gyrs. Here we summarize the history of I $\mathrm{Zw} 18$ age and present an ongoing HST/ACS project which allowed us to precisely derive the galaxy distance by studying its Cepheid variables, and to firmly establish the age of its faintest resolved populations.
\end{abstract}

Keywords. galaxies: dwarf, galaxies: starburst, galaxies: evolution, galaxies: stellar content, galaxies: distances, galaxies: redshifts, galaxies: individual (I Zw 18), (stars: variables:) Cepheids

\section{Introduction}

The blue compact dwarf (BCD) galaxy I Zw 18 is one of the most intriguing nearby objects. Since its discovery by Zwicky (1966), it holds the record of the lowest nebular oxygen abundance of all known star-forming galaxies in the nearby Universe $(12+$ $\log (O / H)=7.2$, corresponding to $1 / 50 \mathrm{Z}_{\odot}$; Skillman \& Kennicutt 1993). This is even more intriguing considering the many efforts devoted over the past 50 years to the search for very metal-poor galaxies at redshift zero. I Zw 18 also has a high gas fraction (e.g., van Zee et al. 1998) and an extremely high star formation (SF) rate per unit mass (Searle \& Sargent 1972) resulting in a blue young stellar population that dominates the integrated luminosity and color. All this observational evidence makes I Zw 18 a chemically unevolved stellar system. At a distance of about $12-15 \mathrm{Mpc}$, it actually represents the closest analog to primordial galaxies in the early universe and it has long been regarded as a possible example of a galaxy undergoing its first burst of star formation.

Many Hubble Space Telescope (HST) studies have focused on the evolutionary state of I Zw 18. The first HST/WFPC2 studies (Hunter \& Thronson 1995; Dufour et al. 1996) 
seemed to confirm that the stars in I Zw 18 are only a few tens of Myr old. From a new improved photometric reduction of the same HST/WFPC2 archival images, our group managed to detect fainter asymptotic giant branch (AGB) stars with ages of at least several hundreds of Myr (Aloisi, Tosi, \& Greggio 1999). These results were confirmed by Östlin (2000) through deep HST/NICMOS imaging. More recently, Izotov \& Thuan (2004) presented new deep HST/ACS imaging observations. Their $I$ vs. $V-I$ CMD shows no sign of an RGB (i.e., low-mass stars with ages $~ 1-13$ Gyr that are burning $\mathrm{H}$ in a shell around a He core) at an assumed distance $D \lesssim 15 \mathrm{Mpc}$. Their conclusion is that the most evolved (AGB) stars are not older than $500 \mathrm{Myr}$ and that I Zw 18 is a bona-fide young galaxy. This result was subsequently challenged by Momany et al. (2005) and our group (Tosi et al. 2006) based on a better photometric analysis of the same data. This showed that many red sources do exist at the expected position of an RGB, and that their density in the CMD drops exactly where a RGB tip (TRGB, at the luminosity of the He flash) would be expected. However, the small number statistics, large photometric errors, and incompleteness, did not allow a more conclusive statement about the possible existence of an RGB in I Zw 18.

The actual nature of I Zw 18 has important cosmological implications. According to hierarchical formation scenarios, dwarf $\left(M \lesssim 10^{9} \mathrm{M}_{\odot}\right)$ galaxies should have been the first systems to collapse and start forming stars. Indeed an RGB has been detected in all metal-poor dwarf irregular galaxies of the Local Group and BCDs within $D \lesssim 15 \mathrm{Mpc}$ that have been imaged with HST (e.g., SBS 1415+437, Aloisi et al. 2005, and references therein). I Zw 18 has remained the only elusive case so far.

The lack of RGB evidence has also made it impossible to pinpoint the distance of I Zw 18 via the TRGB method. Its distance therefore continues to be debated. With a recession velocity of $745 \pm 3 \mathrm{~km} \mathrm{~s}^{-1}$, I Zw 18 has often been assumed to be at a distance of $\sim 10 \mathrm{Mpc}\left(H_{0}=75 \mathrm{~km} \mathrm{~s}^{-1} \mathrm{Mpc}^{-1}\right)$. Correction for Virgocentric infall implies a slightly larger distance between 10 and $14.5 \mathrm{Mpc}(30.0 \lesssim m-M \lesssim 30.8$; Östlin 2000). Izotov et al. (2000) argued that I Zw 18 should be as distant as $20 \mathrm{Mpc}$ to provide consistency between the CMD, the presence of Wolf-Rayet stars and the ionization state of the $\mathrm{H}$ II regions. But they suggested a shorter distance $D \lesssim 15 \mathrm{Mpc}$ from the brightness of AGB stars in Izotov \& Thuan (2004).

\section{New HST/ACS Observations}

We were awarded 24 additional orbits with ACS over a three-month period starting in October 2005 (GO program 10586, PI Aloisi) to better understand the evolutionary status of I $\mathrm{Zw}$ 18. The observations were obtained in 12 different epochs in F606W and F814W to: (1) build a deeper CMD to search for RGB stars; (2) detect and characterize Cepheids at the lowest metallicity available in the local universe; and (3) use both the Cepheids and a possible TRGB detection to determine an accurate distance to I Zw 18 (see Aloisi et al. 2007).

PSF-fitting photometry was performed on deep images that were obtained by combining the exposures in each filter with MultiDrizzle. After application of CTE and aperture corrections, the count rates were transformed to Johnson-Cousins $V$ and $I$ magnitudes. Values shown and discussed hereafter are corrected for $E(B-V)=0.032$ mag of Galactic foreground extinction, but not for any extinction intrinsic to I Zw 18. The archival ACS data in F555W and F814W (GO program 9400, PI Thuan) were also re-processed in a similar manner. The two ACS datasets were then combined using several different approaches and rejection schemes. The results discussed here were obtained by demanding that stars should be detected in all the four deep images ( $V$ and $I$ for both datasets). At 

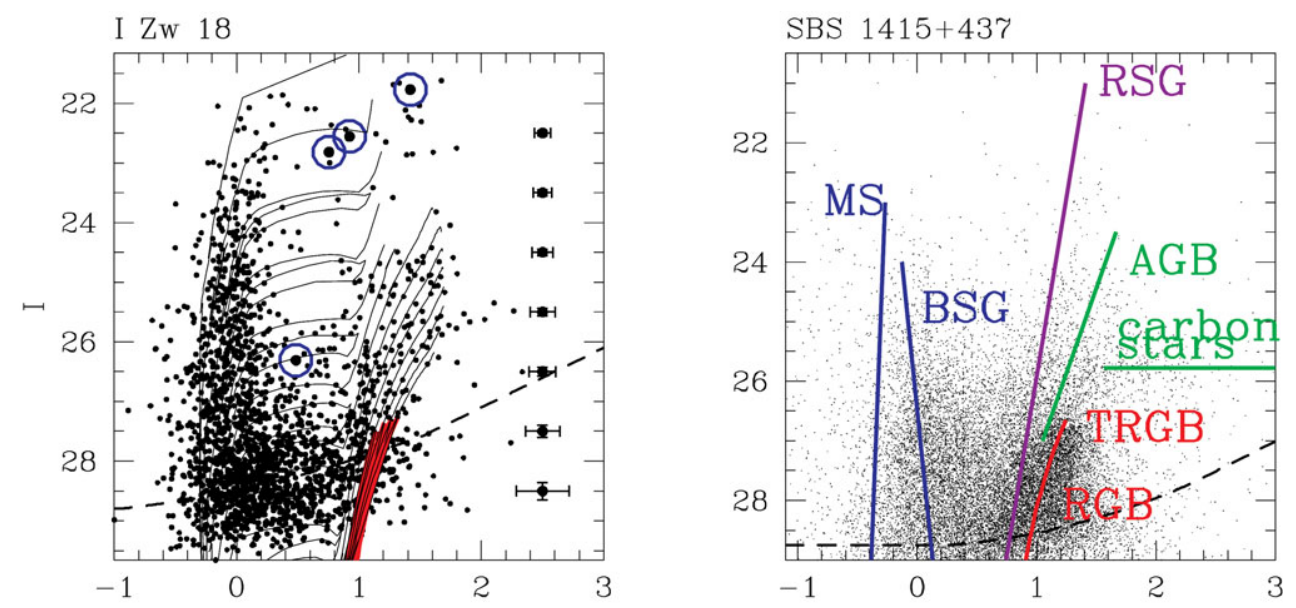

Figure 1. (a) HST/ACS CMD for I Zw 18 (Aloisi et al. 2007). Median photometric errors at $V-I=1$ (determined by comparison of measurements from GO-9400 and GO-10586) are also shown as function of $I$. Padua isochrones from 5.5 Myr to 10 Gyr are overlaid, with the RGB phase for isochrones from 1.7 to $10 \mathrm{Gyr}$ colored red. The isochrones have metallicity $Z=0.0004$ (as inferred from the $\mathrm{H}$ II regions of $\mathrm{I} \mathrm{Zw} 18$ ) and are shown for the distance $D=18.2 \mathrm{Mpc}$ $(m-M=31.30)$. The CMD includes stars in both the main and secondary bodies of I Zw 18 . Blue open circles highlight the four confirmed variables, which are plotted according to their intensity-averaged magnitudes. (b) HST/ACS CMD for SBS $1415+437$ (Aloisi et al. 2005). The main evolutionary sequences seen in the data are indicated in approximate sense as colored straight lines: main sequence (MS), blue supergiants (BSG), red supergiants (RSG), the red giant branch (RGB) with its tip (TRGB), the asymptotic giant branch (AGB), and carbon stars. Both CMDs are corrected for Galactic foreground extinction. Dashed lines are estimates of the $50 \%$ completeness level. The vertical axes of the panels are offset from each other by $0.61 \mathrm{mag}$, i.e. the difference in distance modulus between the galaxies (see Fig. 2). Some $\sim 10$ times more stars were detected in SBS 1415+437, owing to its smaller distance; stars for this galaxy are shown with smaller symbols. The faint red stars in both galaxies indicate that these metal-poor BCD galaxies started forming stars $\gtrsim 1$ Gyr ago.

the expense of some depth, this approach has the advantage of minimizing the number of false detections and therefore providing relatively "clean" CMDs.

\section{Results and Interpretation}

Fig. 1a shows the resulting $I$ vs. $V-I$ CMD of I Zw 18. The CMD shows faint red stars exactly at the position where an RGB would be expected (see the Padua isochrones overplotted in the figure). Figure 2 shows the luminosity function (LF) of the red stars. It shows a sharp drop towards brighter magnitudes, exactly as would be expected from a TRGB. The magnitude of the discontinuity, $I=27.27 \pm 0.14 \mathrm{mag}$, implies a distance modulus $m-M=31.30 \pm 0.17 \mathrm{mag}$ (e.g., Bellazzini et al. 2001), i.e., $D=18.2 \pm 1.5$ Mpc. This assumes that the evolved RGB stars have negligible intrinsic extinction. The TRGB distance is consistent with the distance as inferred from the analysis of the Cepheid variables identified by our program (Aloisi et al. 2007). This agreement further supports our interpretation of the LF drop in Figure 2 as a TRGB feature.

The evidence for an RGB in I Zw 18 is further strengthened by comparison to another BCD, SBS 1415+437, observed by us with a similar HST/ACS set-up (Aloisi et al. 2005). This galaxy is not quite as metal poor as I $\mathrm{Zw} 18(12+\log (O / H)=7.6)$ and is somewhat nearer at $D \approx 13.6 \mathrm{Mpc}$. But taking into account the differences in distance and 


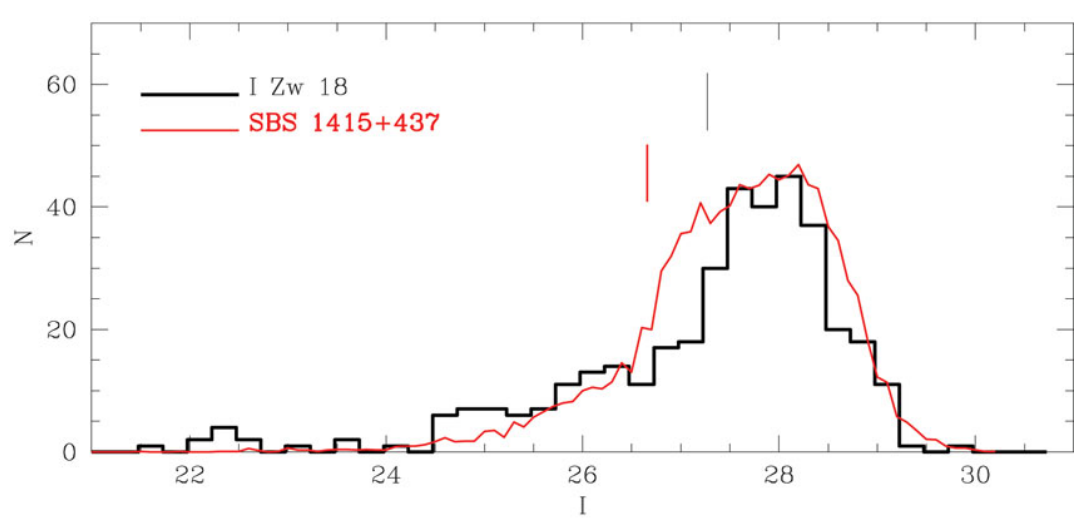

Figure 2. $I$-band LFs for stars with red colors in the range $V-I=0.75-1.5 \mathrm{mag}$, inferred from the CMDs in Fig. 1. Normalizations are arbitrary. Vertical marks indicate the positions of the TRGB, as determined using a Savitzky-Golay filtering technique developed by one of us (see Cioni et al. 2000). At these magnitudes there is a steep LF drop towards brighter magnitudes, due to the end of the RGB sequence. By contrast, the LF drop towards fainter magnitudes at $I>28 \mathrm{mag}$ is due to incompleteness in both cases. Apart from a shift $\Delta(m-M) \approx 0.61$, these metal-poor BCD galaxies have very similar LFs.

completeness, the CMDs of these galaxies look very similar. Since SBS $1415+437$ has an unmistakable RGB sequence, this suggests that such an RGB sequence exists in I Zw 18.

It has been previously suggested that I Zw 18 has no stars with ages $\gtrsim 500$ Myr. It is therefore interesting to ask the question whether there exist Star Formation Histories (SFHs) which do not have stars older than $\sim 1$ Gyr (and by extension, do not have an RGB), yet which can still fit: (1) the observed number of faint red stars in I Zw 18 $(V-I \gtrsim 1.0, I \gtrsim 27.3)$; and $(2)$ the observed LF drop-off at $I \approx 27.3$. Preliminary results of SFH modeling that we have performed indicate that such SFHs do not exist.

\section{Conclusions}

We have obtained new deep HST/ACS observations of I Zw 18 that provide improved insight into the evolutionary status of this benchmark metal-poor BCD. Our results indicate that this galaxy contains RGB stars, in agreement with findings for other local metal-poor BCDs studied with HST. Underlying old ( $\gtrsim 1 \mathrm{Gyr}$ ) populations are therefore present in even the most metal-poor systems. The coherent picture that emerges is that these galaxies did not form recently $(z<0.1)$ and may well be as old as the first systems that collapsed in the early universe. Deeper studies (well below the TRGB) will be needed to pinpoint the exact onset of the star formation in these extreme objects.

We find that at $D=18.2 \pm 1.5 \mathrm{Mpc}, \mathrm{I} \mathrm{Zw} 18$ is more distant than the values $\sim 15 \mathrm{Mpc}$ that have often been assumed in previous work. This may explain why it has remained difficult for so long to unambiguously detect or rule out the presence of old resolved (RGB) stars in this object. The data that we have compiled on Cepheid stars in I Zw 18 will be unique for probing the properties of variable stars at metallicities that have never before been probed.

\section{Acknowledgements}

Support for proposals \#9361 and \#10586 was provided by NASA through a grant from STScI, which is operated by AURA, Inc., under NASA contract NAS 5-26555. 


\section{References}

Aloisi, A., Clementini, G., Tosi, M., Annibali, F., Contreras, R., Fiorentino, G., Mack, J., Marconi, M., Musella, I., Saha, A., Sirianni, M., \& van der Marel, R. P. 2007, ApJ 667, L151

Aloisi, A., Tosi, M., \& Greggio, L. 1999, AJ 118, 302

Aloisi, A., van der Marel, Mack, J., Leitherer, C., Sirianni, M., \& Tosi, M. 2005, ApJ 631, L45

Bellazzini, M., Ferraro, F. R., \& Pancino, E. 2001, ApJ, 556, 635

Cioni, M.-R. L., van der Marel, R. P., Loup, C., \& Habing, H. J. 2000, A\&A, 359, 601

Dufour, R. J., Garnett, D. R., Skillman, E. D., \& Shields, G. A. 1996, in: C. Leitherer, U. Fritzevon Alvensleben, \& J. Huchra (eds.), From Stars to Galaxies: The Impact of Stellar Physics on Galaxy Evolution, Proc. (San Francisco: ASP), p. 358

Hunter, D. A., \& Thronson, H. A. 1995, ApJ 452, 238

Izotov, Y. I., Papaderos, P., Thuan, T. X., Fricke, K. J., Foltz, C., \& Guseva, N. G. 2000, in: A. Weiss, T. G. Abel, \& V. Hill (eds.), The First Stars, Proc. (Berlin: Springer), p. 303

Izotov, Y. I., \& Thuan, T. X. 2004, ApJ 616, 768

Momany, Y., Held, E. V., Saviane, I., Bedin, L. R., Gullieuszik, M., Clemens, M., Rizzi, L., Rich, M. R., \& Kuijken, K. 2005, A\&A 439, 111

Östlin, G. 2000, ApJ 535, L99

Searle, L., \& Sargent, W. L. W. 1972, ApJ 173, 25

Skillman, E. D., \& Kennicutt, R. C. 1993, ApJ 411, 655

Tosi, M., Aloisi, A., Mack, J., \& Maio, M. 2007, in: F. Combes \& J. Palous (eds.), Galaxy Evolution Across the Hubble Time, Proc. IAU Symposium No. 235 (San Francisco: ASP), p. 65

van Zee, L., Westpfahl, D., Haynes, M. P., \& Salzer, J. J. 1998, AJ 115,1000

Zwicky, F. 1966, ApJ 143, 192 